\title{
Whole-lithosphere shear during oblique rifting
}

See more: BrandonLutzGeology.com

Contact: lutz.brandon1@gmail.com

How to rupture a continent?

High strain rate?

High obliquity?

Weak vs strong rheology?
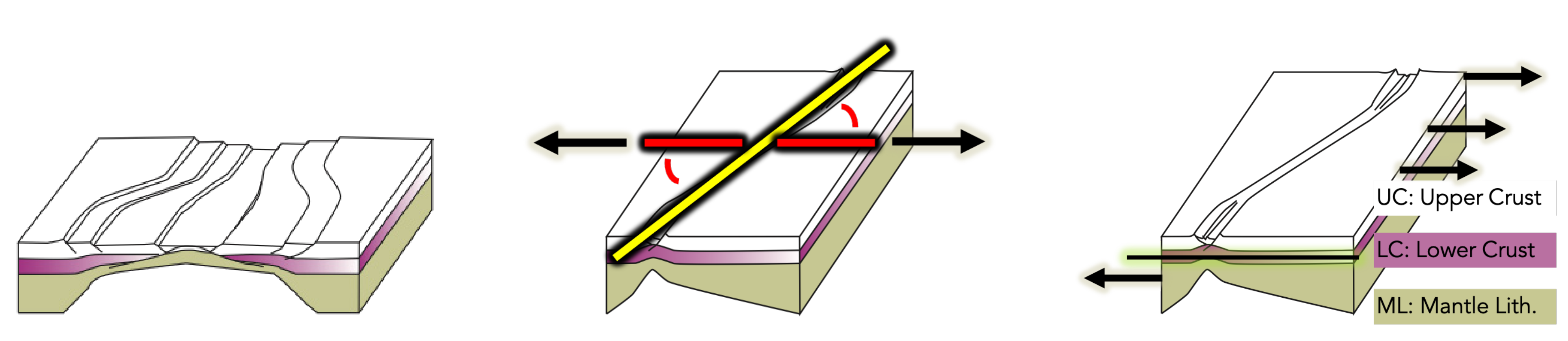

ECSZ / Basin \& Range

Shear zone co-located with MCCs

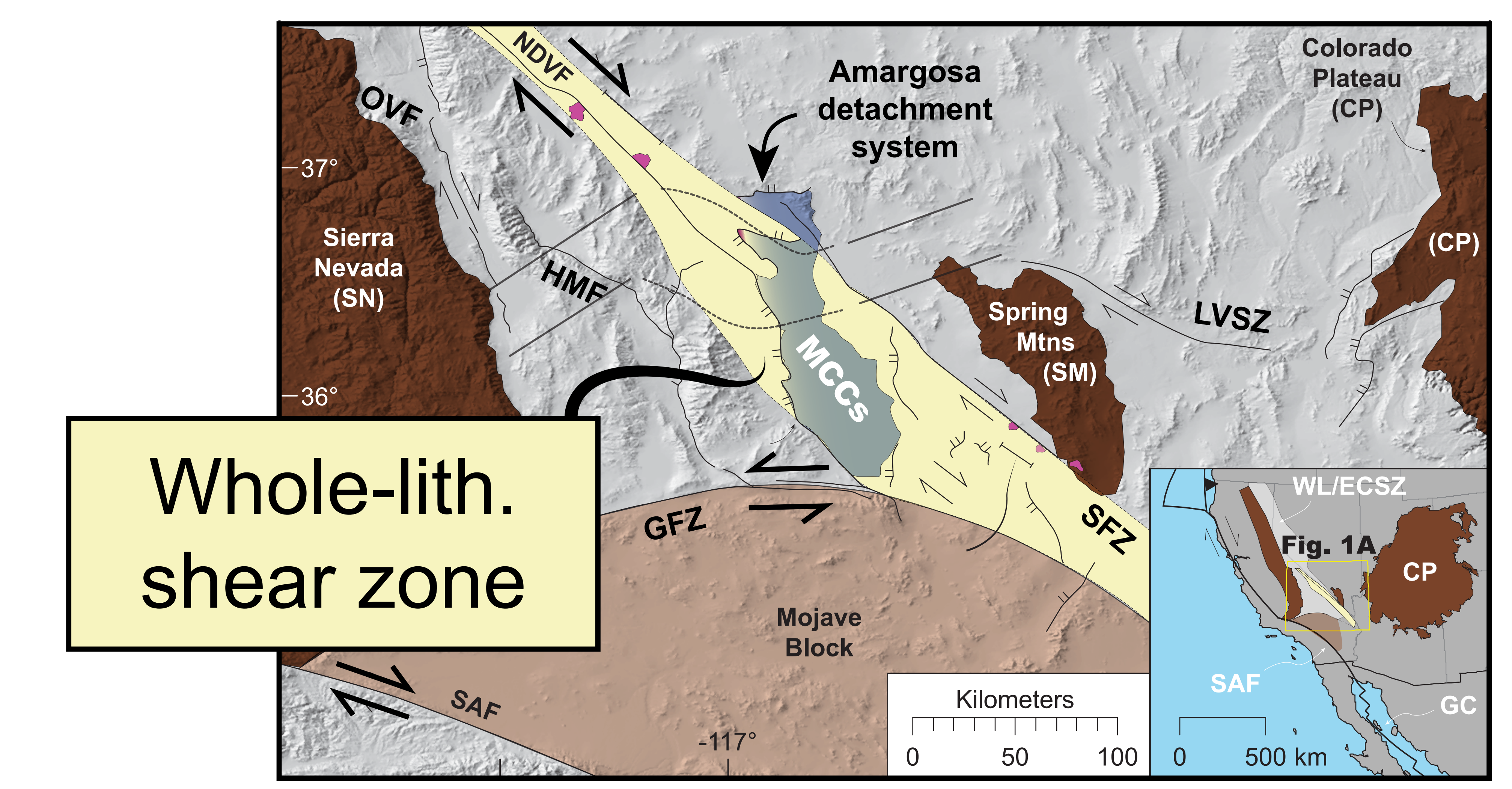

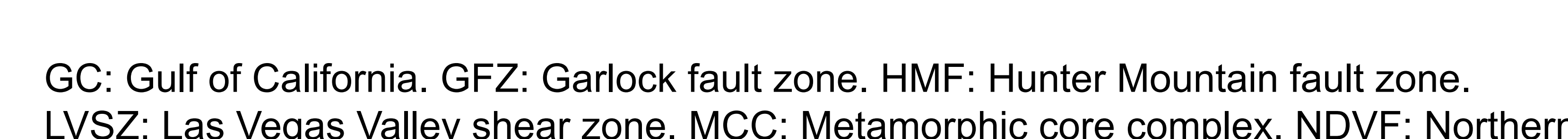

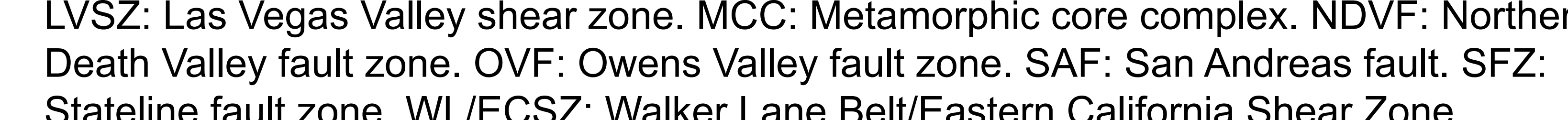

1"'Brandon Lutz, ${ }^{1}$ Gary Axen, ${ }^{1}, 2$ Jolante van Wijk, ${ }^{1}$ Fred Phillips Earth and Environmental Sciences, New Mexico Institute of Mining and Technology, Socorro NM ${ }^{2}$ Earth and Environmental Sciences Division, Los Alamos National Laboratory, Los Alamos, NM ${ }^{*}$ Now at Geological Sciences Department, New Mexico State University, Las Cruces NM

Moho

Reconstruction
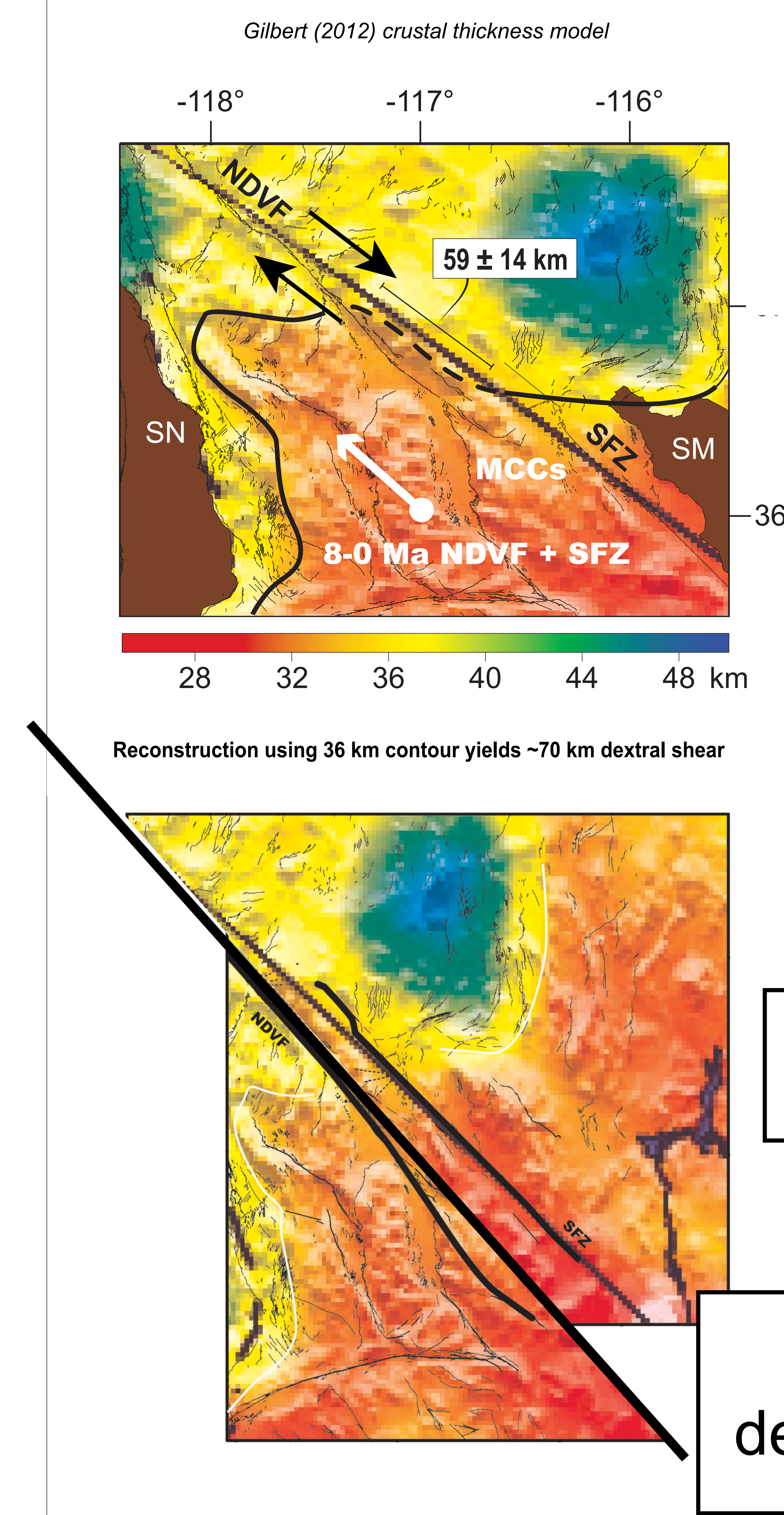

LAB Reconstruction

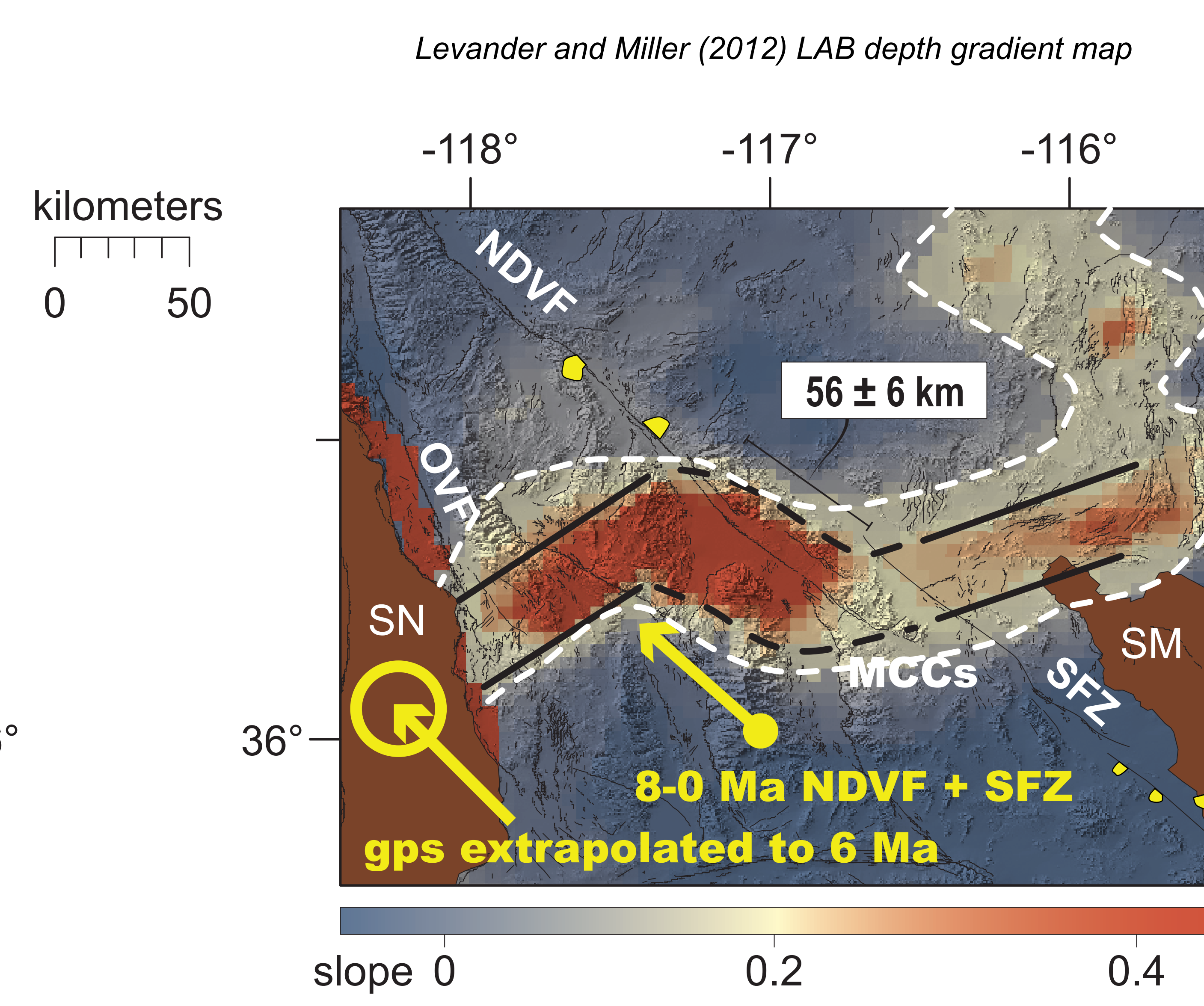
0.2

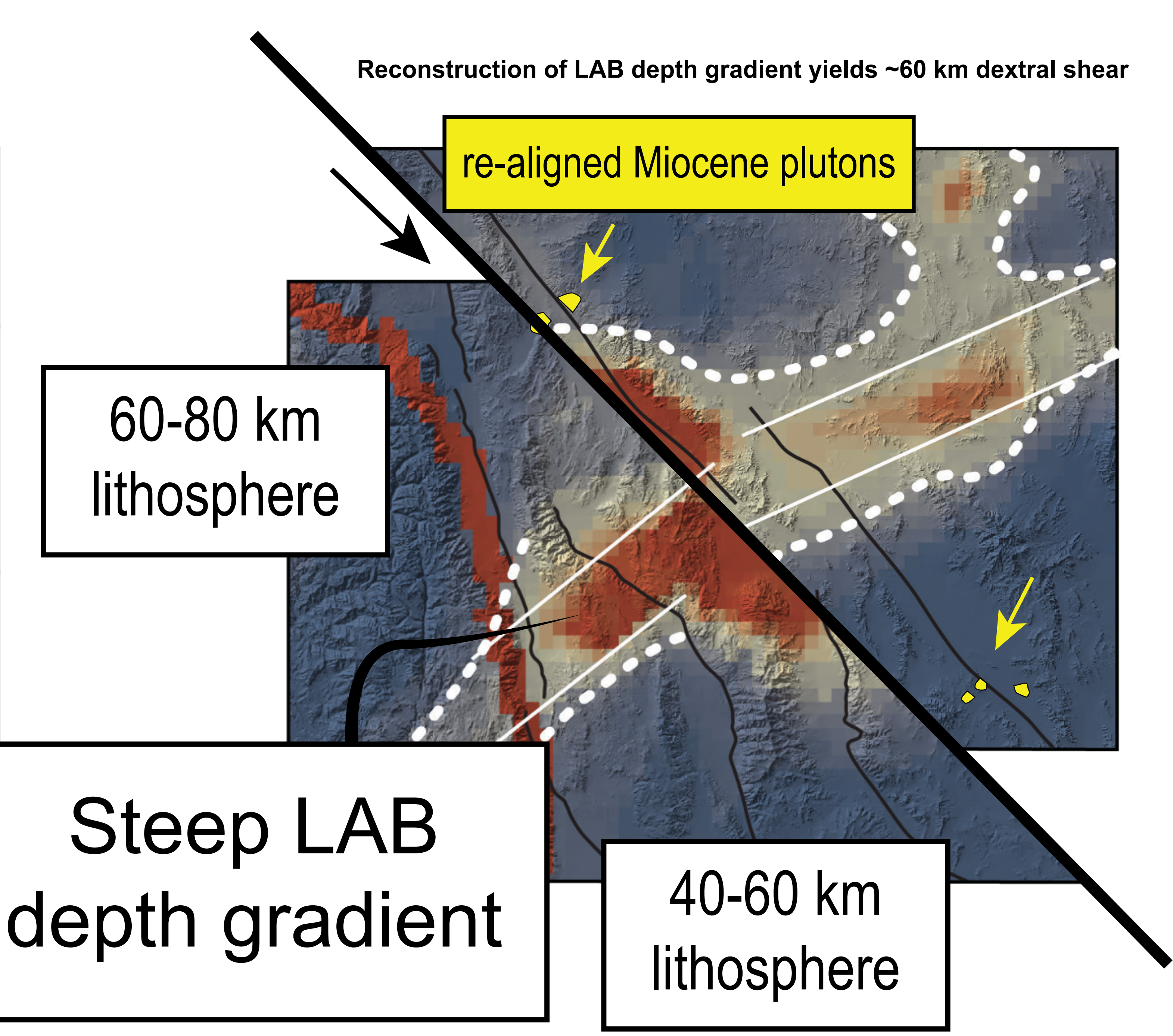

This work was supported by NSF-EAR - 1516680 Adapted from paper in revision to be re-submitted to Geology A (n) Upper Crustal Kinematics
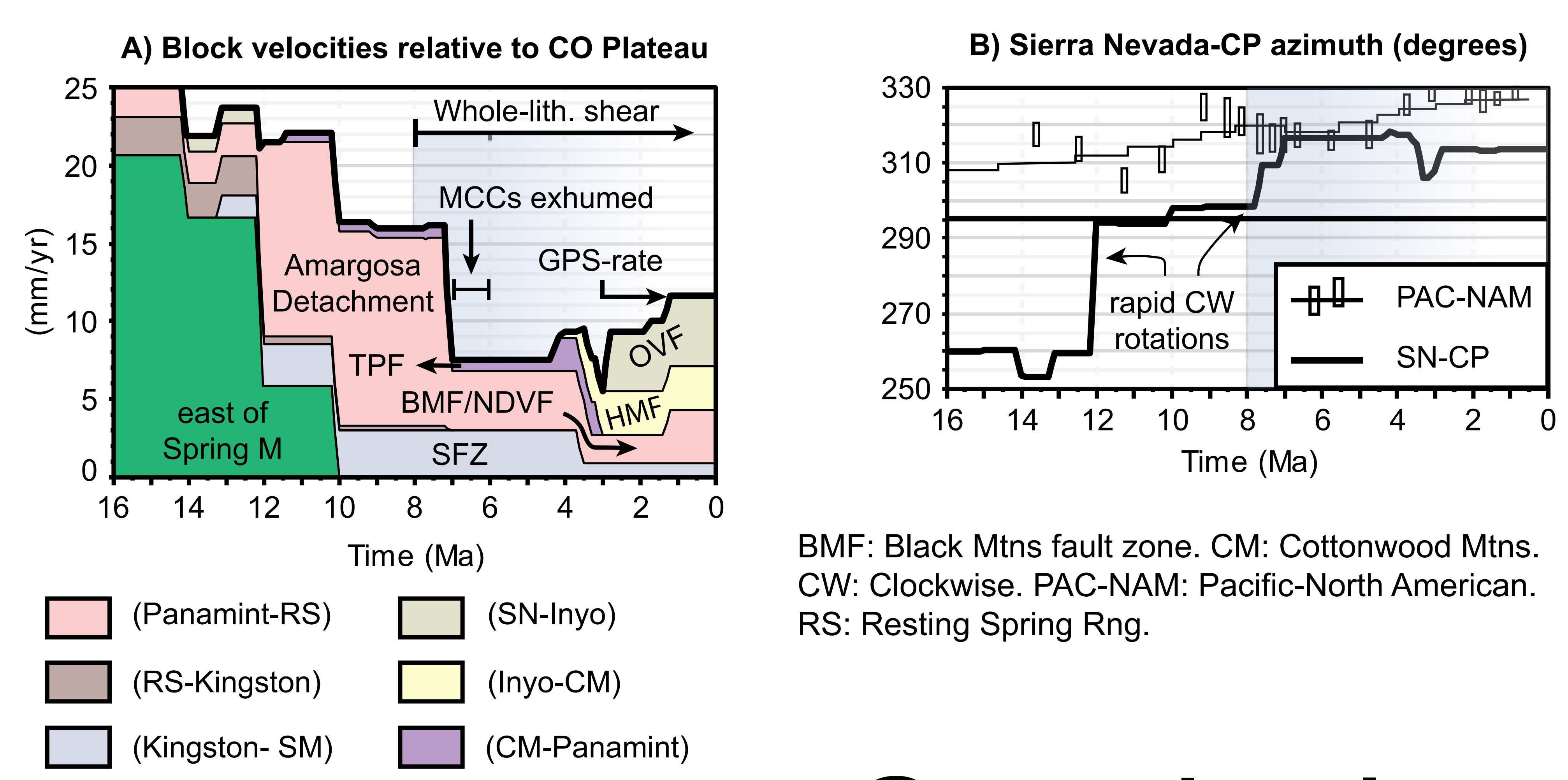

BMFF: Black Mtns fault zone. CM: Cottonwood Mttns.
CW: Clockwise. PAC-NAM: Pacific-North American.

$\square$ (RS-Kingston) $\square$ (nyy-CM)

RS: Resting Spring Rng.

\section{Conclusions}

lithosphere verticallydecoupled by a weak mid-crust

mid-crustal strengthening causes coupling lower crust/mantle

extension direction rotates to become II with

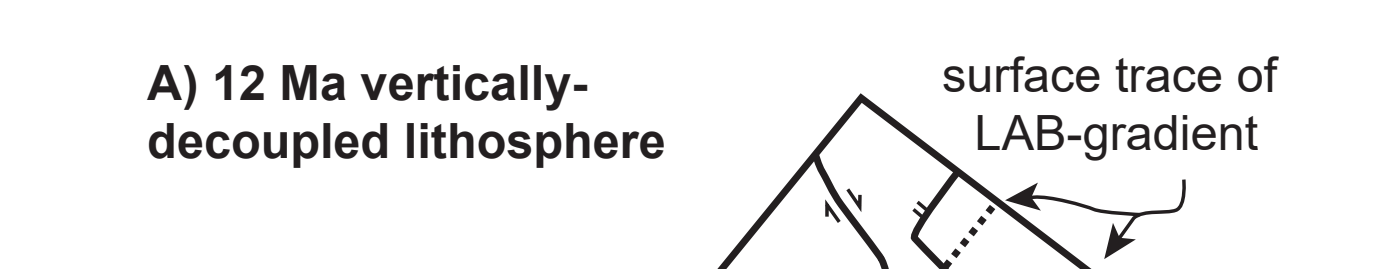
PAC-NAM motion

whole-lithosphere shear beneath MCC belt

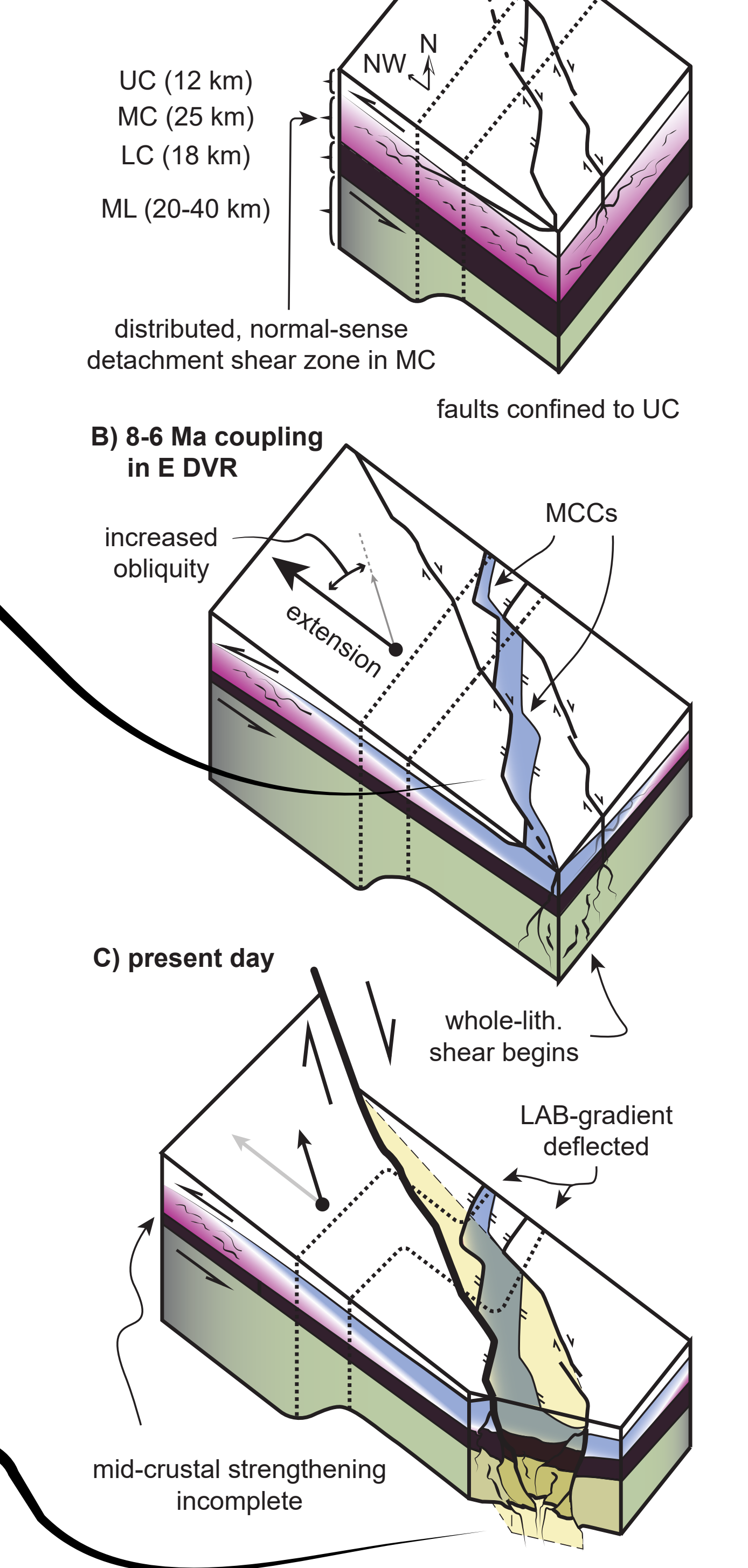

